\title{
Multiphoton antiresonance
}

\author{
M. I. Dykman ${ }^{1}$ and M. V. Fistul ${ }^{2}$ \\ ${ }^{1}$ Department of Physics and Astronomy, Michigan State University, East Lansing, MI 48824, USA \\ 2 Theoretische Physik III, Ruhr-Universität Bochum, D-44801 Bochum, Germany
}

(Dated: August 31, 2018)

\begin{abstract}
We show that nonlinear response of a quantum oscillator displays antiresonant dips and resonant peaks with varying frequency of the driving field. The effect is a consequence of special symmetry and is related to resonant multiphoton mixing of several pairs of oscillator states at a time. We discuss the possibility to observe the antiresonance and the associated multiphoton Rabi oscillations in Josephson junctions.
\end{abstract}

PACS numbers: 05.45.-a, 05.60.Gg, 74.50.+r, 33.80.Wz

Many resonant nonlinear phenomena are described by the model of a nonlinear oscillator in a resonant field. Examples include collisionless dissociation of molecules [1], dispersive optical bistability [2], cyclotron resonance of a relativistic electron 3], resonant enhancement of activated escape [4] and tunneling [5] in Josephson junctions, and recently discovered hysteresis in Josephson junctions [6] and nanomechanical resonators [].

A weakly nonlinear oscillator is a multi-level quantum system with nearly equidistant energy levels $E_{n}$. Therefore a periodic force of frequency $\omega_{F}$ can be nearly resonant for many transitions at a time, i.e., $\hbar \omega_{F}$ can be close to the interlevel distance $E_{n+1}-E_{n}$ for many $n$. This makes an oscillator convenient for studying multiphoton Rabi oscillations. They arise when the spacing between remote energy levels $n$ and $m$ coincides with the energy of $n-m$ photons, $E_{n}-E_{m}=(n-m) \hbar \omega_{F}$ 1]. The multiphoton transition amplitude is resonantly enhanced, because the $m \rightarrow n$ transition occurs via a sequence of virtual field-induced transitions $k \rightarrow k+1$ (with $m \leq k \leq n-1$ ), all of which are almost resonant.

In this paper we show that multiphoton transitions in the oscillator are accompanied by a new effect, an antiresonance of the response. When the frequency of the driving field adiabatically passes through a resonant value, the vibration amplitude displays a sharp minimum or maximum, depending on the initial conditions. We argue that the antiresonance and the multiphoton Rabi oscillations can be observed in such macroscopic systems as Josephson junctions and nanomechanical resonators.

The multiphoton antiresonance is a consequence of two interesting properties of a driven oscillator. First, for resonant photon frequencies, simultaneously, not one but several pairs of states turn out to be in resonance. Second, the amplitudes of forced vibrations in the resonating states coincide with each other, in the neglect of multiphoton mixing. For not too strong fields, these amplitudes are determined by nonresonant field-induced coupling of neighboring Fock states of the oscillator. The resonant multiphoton mixing leads to level splitting, which strongly differs for different resonating pairs. It is this difference that results in the dips (peaks) in the vibration amplitude as $\omega_{F}$ adiabatically passes through resonances.

In the semiclassical picture, resonant multiphoton transitions correspond to tunneling between Floquet states of the oscillator with equal quasienergies [the quasienergy $\varepsilon$ gives the change of the wave function $\psi(t)$ when time is incremented by the modulation period $\tau_{F}$, $\left.\psi\left(t+\tau_{F}\right)=\exp \left(-i \varepsilon \tau_{F} / \hbar\right) \psi(t)\right]$. The occurrence of equalquasienergy states is related to the bistability of forced vibrations of a classical oscillator [8]. There is similarity between the oscillator tunneling and the tunneling of a particle in a static double-well potential, with the potential minima being analogs of the stable states of forced vibrations. However, the latter states are not separated by a static barrier. Tunneling of a driven oscillator [9] is a carefully studied example of dynamical tunneling [10]. As we show, the WKB analysis gives an important insight into the origin of the antiresonance.

The Hamiltonian of a driven nonlinear oscillator with mass $M=1$ has the form

$$
H(t)=\frac{1}{2} p^{2}+\frac{1}{2} \omega_{0}^{2} q^{2}+\frac{1}{4} \gamma q^{4}-q A \cos \left(\omega_{F} t\right) .
$$

We assume that the driving field is nearly resonant, i.e., the frequency detuning $\delta \omega$ is small,

$$
|\delta \omega| \ll \omega_{F}, \quad \delta \omega=\omega_{F}-\omega_{0} .
$$

We consider not too large amplitudes of the driving field $A$, so that the oscillator anharmonicity is small, and in particular $|\gamma| q^{2} \ll \omega_{0}^{2}$ for typical $q$. We also assume that $\gamma$ and $\delta \omega$ have the same sign. This is required for the amplitude of classical forced vibrations to display hysteresis as a function of the force amplitude $A$. If there is a cubic term $\alpha q^{3} / 3$ in the potential energy of the oscillator, its major effect of interest for this paper is the renormalization $\gamma \rightarrow \gamma-\frac{10}{9}\left(\alpha / \omega_{0}\right)^{2}$ [8].

To study quantum dynamics, we will write the Hamiltonian in terms of the raising and lowering operators of the oscillator $a^{\dagger}, a$, and switch to the rotating frame with a canonical transformation $U(t)=\exp \left(-i \omega_{F} a^{\dagger} a t\right)$. The transformed Hamiltonian $H_{0}=U^{\dagger}(t) H(t) U(t)-$ $i \hbar U^{\dagger}(t) \dot{U}(t)$ is time-independent in the rotating wave approximation (RWA),

$$
\begin{gathered}
H_{0}=-\delta \omega \hat{n}+\frac{1}{2} V \hat{n}(\hat{n}+1)-f\left(a+a^{\dagger}\right), \quad \hat{n}=a^{\dagger} a \\
V=3 \hbar \gamma / 4 \omega_{0}^{2}, \quad f=\left(8 \hbar \omega_{0}\right)^{-1 / 2} A
\end{gathered}
$$


The terms $\propto V, f$ that contain fast-oscillating factors $\propto$ $\exp \left( \pm i k \omega_{F} t\right)$ with $k=2,4$ were disregarded in Eq. (3). In the expression for $H_{0}$ and in what follows $\hbar=1$.

The eigenvalues of the Hamiltonian (3) $\varepsilon_{n}$ give the quasienergies of the driven oscillator. In the limit of weak driving their spectrum is particularly simple,

$$
\varepsilon_{n}=-n \delta \omega+V n(n+1) / 2 \quad(f \rightarrow 0) .
$$

We will study multiphoton resonance for the ground state of the oscillator, $E_{N}-E_{0}=N \omega_{F}$, or equivalently $\varepsilon_{0}=\varepsilon_{N}$. From Eq. (4), for small $f$ and given $N$ the resonance occurs for

$$
\delta \omega=\delta \omega_{N} \equiv V(N+1) / 2 .
$$

Remarkably, at resonance all quasienergy levels (4) with $n \leq N$ are pairwise-degenerate, $\varepsilon_{N-n}=\varepsilon_{n}$ [11]. Equivalently, $E_{N-n}-E_{n}=(N-2 n) \omega_{F}$. One can see that the degeneracy is not lifted by the lowest-order $\left(\propto f^{2}\right)$ fieldinduced level shift [except for the levels $n=(N \pm 1) / 2$ for odd $N$ and $n=(N / 2) \pm 1$ for even $N]$. As shown below, it persists for all $f$ in the WKB approximation in the neglect of tunneling.

The response of the oscillator to the field is characterized by the expectation value of its coordinate $q$. If the oscillator is in an eigenstate $|n\rangle$ of the Hamiltonian (3),

$$
q_{n}=\left(2 \omega_{0}\right)^{-1 / 2} a_{n} e^{-i \omega_{F} t}+\text { c.c., } \quad a_{n}=\langle n|a| n\rangle .
$$

To first order in the field, the reduced amplitude of forced vibrations $a_{n}$ is

$$
a_{n}=-f \delta \omega /((\delta \omega-V n)[\delta \omega-V(n+1)]) .
$$

At multiphoton resonance, where $\delta \omega=\delta \omega_{N}$, the vibration amplitudes in the resonating states coincide with each other, $a_{N-n}=a_{n}$ for $0 \leq n<N / 2$.

Multiphoton mixing leads to splitting of the quasienergy levels and the vibration amplitudes. It can be calculated by diagonalizing the Hamiltonian (3) and is shown in Fig. Mas a function of frequency detuning $\delta \omega$. One of the involved resonating states is the ground state of the oscillator $n=0$ in the limit $f \rightarrow 0$.

The minimal splitting of the levels $\varepsilon_{0}$ and $\varepsilon_{N}$ is given by the multiphoton Rabi frequency $\Omega_{R}$. For weak field it can be obtained from Eq. (3) by perturbation theory [1]. To the lowest order in $f / \delta \omega_{N}$

$$
\Omega_{R}=2 f|2 f / V|^{N-1} N^{2}(N !)^{-3 / 2} .
$$

For $N \gg 1$ this expression becomes

$$
\begin{aligned}
& \Omega_{R}=V\left(f / f_{N}\right)^{N} N^{5 / 4}(2 \pi)^{-3 / 4}, \\
& f_{N}=|V| N^{3 / 2} \exp (-3 / 2) / 2 .
\end{aligned}
$$

The Rabi frequency depends on $N$ exponentially, $\Omega_{R} \propto$ $f^{N}$. In the case $N=5$ it is shown in Fig. 2]. One can see from this figure that Eq. (7) works well in the whole range of the field amplitudes $f / f_{N} \lesssim 0.5$. For larger
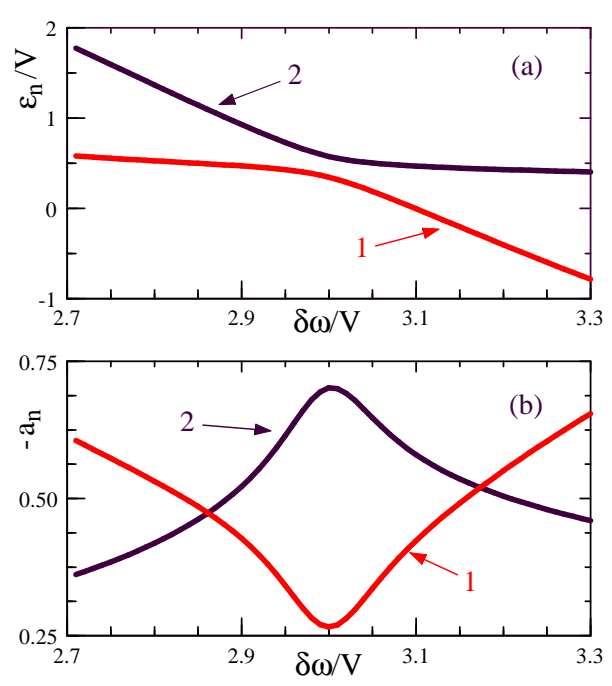

FIG. 1: (a) Anticrossing of the quasienergy levels $\varepsilon_{0}$ and $\varepsilon_{5}$ with varying frequency $\delta \omega$ for the field amplitude $f / f_{5}=0.75$, where the scaling field $f_{5}$ is given by Eq. (8) with $N=5$. In the limit $f \rightarrow 0$ the levels cross for $\delta \omega=\delta \omega_{5}=3 V$; (b) The reduced vibration amplitudes $a_{0}$ and $a_{5}$ for the same adiabatic states. For $\left(\delta \omega_{5}-\delta \omega\right) / \Omega_{R} \gg 1$ the states described by lines 1 and 2 are close to the Fock states of the oscillator $|0\rangle$ and $|5\rangle$, respectively.

fields $\Omega_{R}$ depends on $f$ much weaker than the asymptotic expression (8) [9].

The most interesting feature of Fig. 1 is the antiresonant splitting of the amplitudes. It occurs at the adiabatic passage of $\delta \omega$ through resonance, where the system switches between the ground and excited states. In particular, the amplitude displays an antiresonant dip if the oscillator is mostly in the ground state for $\left(\delta \omega-\delta \omega_{N}\right) / V<1$ or in the state $N$ for $\left(\delta \omega-\delta \omega_{N}\right) / V>1$. The magnitude and sharpness of the dip are determined by $\Omega_{R} / V$ and depend very strongly on the field and $N$. With decreasing $\Omega_{R} / V$ the dip (and peak) start looking like cusps located at resonant frequency. The amplitude splitting as function of the field is shown in Fig. 2

To explain this behavior we note that, for $\delta \omega=\delta \omega_{N}$, the field leads to two major effects. One is resonant mixing of the wave functions into symmetric and antisymmetric combinations $|n, N-n\rangle_{ \pm}=\left(|n\rangle_{0} \pm|N-n\rangle_{0}\right) / \sqrt{2}$ with quasienergies $\varepsilon_{n \pm}\left(|\cdot\rangle_{0}\right.$ are eigenfunctions of the operator $\hat{n}=a^{\dagger} a$ ). The second effect is nonresonant mixing of the states $|n, N-n\rangle_{ \pm}$with different $n$, which leads to nonzero expectation values of the vibration amplitudes.

To first order in $f$, the vibration amplitudes $a_{0 \pm}=$ ${ }_{ \pm}\langle 0, N|a| 0, N\rangle_{ \pm}$are determined by nonresonant mixing of the states $|0, N\rangle_{ \pm}$with $|1, N-1\rangle_{ \pm}$and $|N+1\rangle_{0}$. For comparatively weak fields, the level splitting $\varepsilon_{1+}-\varepsilon_{1-} \propto$ $\Omega_{R}(\delta \omega / f)^{2}$ largely exceeds the splitting $\varepsilon_{0+}-\varepsilon_{0-}=\Omega_{R}$. Then from perturbation theory $a_{0+}-a_{0-} \propto f\left[\left(\varepsilon_{0}-\right.\right.$ $\left.\left.\varepsilon_{1+}\right)^{-1}-\left(\varepsilon_{0}-\varepsilon_{1-}\right)^{-1}\right] \propto(f / V)^{N-1}$. This scaling describes the resonant small-field amplitude splitting in 

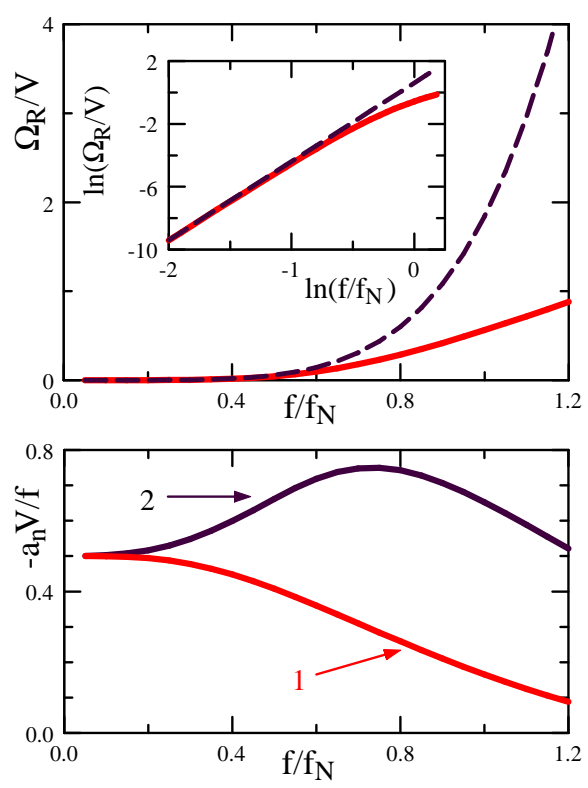

FIG. 2: Upper panel: field-induced splitting of the quasienergy levels $n=0$ and $n=N=5$ for resonant driving frequency, $\delta \omega=\delta \omega_{5}=3 V$. The splitting gives the 5 -photon Rabi frequency $\Omega_{R}$. The dashed line shows the weak-field perturbation theory (7). Lower panel: splitting of the reduced amplitudes of forced vibrations in the corresponding Floquet states. The curve labelling coincides with that in Fig. 1]

Fig. 2 extremely well (the prefactor is determined by the admixture of states $|n, N-n\rangle_{ \pm}$with $n>1$ and will be discussed elsewhere).

The simultaneous degeneracy of quasienergies and vibration amplitudes for many pairs of states in a broad field range can be shown analytically in the case where the oscillator dynamics is described by the WKB approximation. This approximation applies for

$$
\lambda \ll 1, \quad \lambda=V /(2 \delta \omega) .
$$

It is convenient to introduce the reduced coordinate and momentum of the oscillator in the rotating frame

$$
Q=(V / 4 \delta \omega)^{1 / 2}\left(a+a^{\dagger}\right), \quad P=-i(V / 4 \delta \omega)^{1 / 2}\left(a-a^{\dagger}\right)
$$

with the commutator $[P, Q]=-i \lambda$. In these variables, in the neglect of terms $\propto \lambda$, the Hamiltonian (3) becomes $H_{0}=2(\delta \omega)^{2} V^{-1}[g(Q, P)-1 / 4]$, where

$$
g(Q, P)=\left(Q^{2}+P^{2}-1\right)^{2} / 4-\beta^{1 / 2} Q .
$$

Here $\beta=f^{2} V /(\delta \omega)^{3}$ is the reduced field intensity.

The function $g(Q, P)$ is illustrated in Fig. 3. $\delta \omega g(Q, P)$ is the classical Hamiltonian in the RWA, it gives the quasienergy of the oscillator [9, 12]; $Q, P$ are the canonical variables. The minimum and local maximum of $g(Q, P)$ correspond to the stable states of forced vibrations. They coexist for $0<\beta<4 / 27$. For such $\beta$, in a certain range of $g$ there are two Hamiltonian trajectories with the same $g$, one on the internal "dome" and the other on the external part of the surface $g(Q, P)$. We call them, respectively, internal and external trajectories.

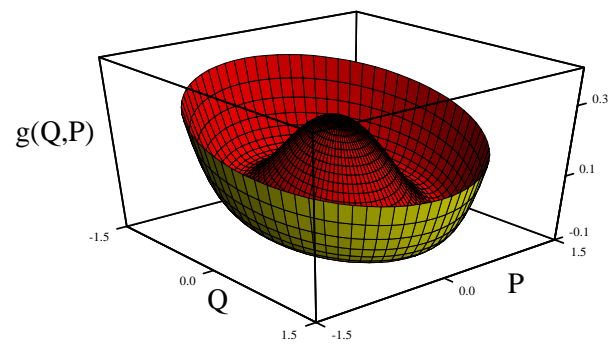

FIG. 3: The classical quasienergy of the oscillator (10). The plot refers to the reduced field $\beta=2 / 27$.

The external trajectory for given $g(Q, P)=g$ has the form $Q(t)=\beta^{-1 / 2}\left[X^{2}(t)-g\right]$, with

$$
X(t)=\frac{c_{2}\left(c_{1}-c_{3}\right)-c_{3}\left(c_{1}-c_{2}\right) \mathrm{sn}^{2} u}{c_{1}-c_{3}-\left(c_{1}-c_{2}\right) \operatorname{sn}^{2} u}
$$

Here, sn $u$ is the Jacobi elliptic function; the elliptic modulus is $m=\left(c_{1}-c_{2}\right)\left(c_{3}-c_{4}\right) /\left(c_{1}-c_{3}\right)\left(c_{2}-c_{4}\right)$, and $u=\left[\left(c_{1}-c_{3}\right)\left(c_{2}-c_{4}\right)\right]^{1 / 2} \delta \omega t / 2$ is the appropriately scaled time. The coefficients $c_{1}>c_{2}>c_{3}>c_{4}$ are the roots of the polynomial $\beta(1+2 x)-\left(x^{2}-g\right)^{2}\left(X=c_{1}, c_{2}\right.$ and $X=c_{3}, c_{4}$ gives the turning points $\dot{Q}=0$ on the external and internal trajectories, respectively).

The internal trajectory $Q(t)$ is given by Eq. (11) with $u \rightarrow u+K+i K^{\prime}$, where $K \equiv K(m)$ is the complete elliptic integral, and $K^{\prime} \equiv K(1-m)$.

An immediate consequence of the analytical interrelation between the external and internal trajectories is that the periods of motion along them coincide [13]. The vibration frequency is $\omega(g)=\pi\left[\left(c_{1}-c_{3}\right)\left(c_{2}-c_{4}\right)\right]^{1 / 2} \delta \omega / 2 K$. When motion is quantized, $\omega(g)$ gives the distance between the energy levels. Therefore if, for some $\delta \omega$ and $\beta$, two levels that correspond to the external and internal trajectories coincide with each other, many levels will coincide pairwise as well. Resonant multiphoton splitting of the levels is due to tunneling between the external and internal parts of the surface $g(Q, P)$.

In the WKB approximation the expectation value $a_{n}$ in a quantum state $|n\rangle$ is given by the period-averaged value $\langle Q\rangle_{g}$ of the coordinate $Q$ on the appropriate classical trajectory. The values of $\langle Q\rangle_{g}$ turn out to be the same on the internal and external trajectories with the same $g$. Indeed, their difference can be written as a contour integral of $X^{2}$ in the $u$-plane. The contour is a parallelogram with vertices at $u=0,2 K, 3 K+i K^{\prime}$, and $K+i K^{\prime}$. The function $X^{2}$ has one second order pole in this parallelogram. The residue is $\propto c_{1}+c_{2}+c_{3}+c_{4}=0$. This proves that the vibration amplitudes of the states with equal quasienergies are equal, in the neglect of tunneling.

In order to observe the coherent multiphoton quantum effects, the Rabi frequency $\Omega_{R}$ should exceed the decoherence rate. In the RWA, relaxation of an oscillator can 
often be described by the master equation for the density matrix $\rho$. In many cases of interest the major source of decoherence is noise or a quantum field that modulates the oscillator energy. Then the coupling operator is $\propto \hat{n}$. If the modulation is fast compared to $\delta \omega, V$, the master equation takes the form

$$
\dot{\rho}=i\left[\rho, H_{0}\right]-\hat{\Gamma}_{\varphi} \rho, \quad \hat{\Gamma} \rho=\Gamma_{\varphi}[\hat{n},[\hat{n}, \rho]],
$$

where $\Gamma_{\varphi}$ characterizes the noise intensity. It gives the phase diffusion coefficient of the oscillator.

From Eq. (12), for resonant $\delta \omega=\delta \omega_{N}$ the major effect of phase diffusion is decay of the Rabi oscillations. For $\Omega_{R} / \delta \omega_{N} \ll 1$ the decay rate is $\Gamma_{\varphi} N^{2}$ : this is simply the diffusion coefficient of the phase difference of the Fock states of the oscillator $|0\rangle$ and $|N\rangle$. More formally, this is the decay rate of the difference $\rho_{++}-\rho_{--}$, where $\rho_{\nu \nu^{\prime}}=$ ${ }_{\nu}\langle 0, N|\rho| 0, N\rangle_{\nu^{\prime}}\left(\nu, \nu^{\prime}= \pm\right)$.

The decoherence rate due to phase diffusion quickly increases with $N$. Our results demonstrate that the strong resonant amplitude splitting occurs already for $N=5$. We emphasize that this is a coherent quantum effect. It is qualitatively different from the nonmonotonic field dependence of the stationary amplitude of a driven damped oscillator for nonzero temperatures [14, 15].

The antiresonances in the vibration amplitude can be directly observed in Josephson junctions (JJ's), which are well described by the model of a nonlinear oscillator (3). The amplitude of forced vibrations of a JJ was recently measured and the vibration bistability was demonstrated 6]. The eigenfrequency and nonlinearity of a JJ are controlled by the dc bias current $I_{\mathrm{dc}}$. When $I_{\mathrm{dc}}$ is close to the critical current $I_{0}$, i.e., $\eta=\left(I_{0}-I_{\mathrm{dc}}\right) / I_{0} \ll 1$, in Eq. (3) $\omega_{0}=\omega_{p}(2 \eta)^{1 / 4}$ and $V=-5 \hbar \omega_{p}^{2} / 48 E_{J} \eta$, where $\omega_{p}$ is the JJ plasma frequency and $E_{J}=\hbar I_{0} / 2 e$. The scaling RF current $I_{N}$ for a resonant $N$-photon transition, which corresponds to the scaling field $f_{N}$ in Eq. (8), is

$$
I_{N}=(5 / 48) I_{c} \exp (-3 / 2)\left(2 N \hbar \omega_{p} / E_{J}\right)^{3 / 2}(2 \eta)^{-7 / 8} .
$$

For the RF current $I_{\mathrm{RF}} \sim I_{N}$ at resonant frequency $\omega_{F}=\omega_{0}+V(N+1) / 2$, the splitting of the vibration amplitude is strong, see Figs. 11 2] Roughly, $I_{N}$ is inversely proportional to $I_{0}-I_{\mathrm{dc}}$, as is also the distance $V / 2$ between the resonant values $\omega_{F}$.

Multiphoton Rabi oscillations in multilevel JJs can be also studied by measuring the rate of tunneling decay induced by resonant RF pulses of different length $t_{\mathrm{RF}}$. The effective potential of a JJ is metastable, $\omega_{0}^{2} q^{2} / 2-\alpha q^{3} / 3$. The probability of tunneling escape from an excited state is much larger than from the ground state, and the higher the energy level the stronger is the difference. Therefore at multiphoton resonance the escape rate should oscillate as $\sin ^{2}\left(\Omega_{R} t_{\mathrm{RF}} / 2\right)$. This approach has been used to detect single-photon Rabi oscillations in strongly nonlinear JJ's that have a small number of metastable states [16].

We note that the quantum effects that we discuss can be studied also in nanomechanical systems where classical bistability of forced vibrations was observed [7].

In this paper we have shown that multiphoton response of a quantum oscillator may display antiresonant dips (peaks) as a function of frequency. The effect is due to level anticrossing and coherent mixing of many oscillator states at a time. The shape and magnitude of the dips (peaks) strongly depend on the field. We find the multiphoton Rabi frequency and discuss the possibility to observe the antiresonant response and multiphoton Rabi oscillations in Josephson junctions and nanomechanical resonators.

The work of M.D. was supported in part by the NSF through grant No. ITR-0085922. M. V. F. thanks the hospitality of Michigan State University where this work was started, and the financial support of SFB 691.
[1] D.M. Larsen and N. Bloembergen, Opt. Communications 17, 254 (1976)

[2] P. D. Drummond and D. F. Walls, J. Phys. A 13, 725 (1980).

[3] G. Gabrielse, H. Dehmelt, and W. Kells, Phys. Rev. Lett. 54, 537 (1985).

[4] A.I. Larkin and Yu.N. Ovchinnikov, J. Low Temp. Phys. 63, 317 (1986); B.I. Ivlev and V.I. Mel'nikov, Phys. Lett. A 116, 427 (1986); S. Linkwitz and H. Grabert, Phys. Rev. B 44 11888, 11901 (1991); M.H. Devoret et al., Phys. Rev. B 36, 58-73 (1987).

[5] A. Wallraff et al. Phys. Rev. Lett. 90, 037003 (2003); M. V. Fistul, A. Wallraff, and A. V. Ustinov, Phys. Rev. B, 68, 060504(R) (2003).

[6] I. Siddiqi et al., cond-mat/0312623

[7] J. S. Aldridge and A. N. Cleland, cond-mat/0406528

[8] L.D. Landau and E.M. Lifshitz Mechanics (Pergamon, London 1976).
[9] A.P. Dmitriev and M.I. D'yakonov, Zh. Eks. Teor. Fiz. 90, 1430 (1986) [Sov. Phys. JETP 63, 838 (1986)].

[10] M. J. Davis and E. J. Heller, J. Chem Phys. 75, 246 (1981).

[11] H. Risken et al., Phys. Rev. A 35, 1729 (1987).

[12] M.I. Dykman and M.A. Krivoglaz, Zh. Eksp. Teor. Fiz. 77, 60 (1979) [Sov. Phys. JETP 50, 30 (1979).

[13] M.I. Dykman and V.N. Smelyanskiy, Zh. Eksp. Teor. Fiz. 94, 61 (1988) [Sov. Phys. JETP 67, 1769 (1988)]

[14] K. Vogel and H. Risken, Phys. Rev. A 38, 2409 (1988).

[15] V. Peano and M. Thorwart, cond-mat/0407720

[16] J. R. Friedman et al., Nature 406, 43 (2000); C. H. van der Wal et al., Science, 290, 773 (2000); J. M. Martinis et al., Phys. Rev. Lett. 89, 117901 (2002); Y. Yu et al., Science, 296, 889 (2002); A.J. Berkley et al., Phys. Rev. B 68, 060502(R) (2003). 